WellBeing International

WBI Studies Repository

$10-2000$

\title{
Relative Volume of the Cerebellum in Dolphins and Comparison with Anthropoid Primates
}

L. Marino

Emory University

James K. Rilling

Emory University

Shinko K. Lin

Emory University

Sam H. Ridgway

Navy Marine Mammal Program

Follow this and additional works at: https://www.wellbeingintlstudiesrepository.org/acwp_vsm

Part of the Animal Structures Commons, Animal Studies Commons, and the Veterinary Anatomy

Commons

\section{Recommended Citation}

Marino, L., Rilling, J. K., Lin, S. K., \& Ridgway, S. H. (2000). Relative volume of the cerebellum in dolphins and comparison with anthropoid primates. Brain Behavior and Evolution, 56(4), 204-211.

This material is brought to you for free and open access by WellBeing International. It has been accepted for inclusion by an authorized administrator of the WBI Studies Repository. For more information, please contact wbisr-info@wellbeingintl.org.

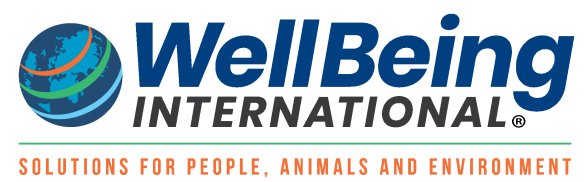




\title{
Relative Volume of the Cerebellum in Dolphins and Comparison with Anthropoid Primates
}

\author{
Lori Marino ${ }^{1}$, James K. Rilling ${ }^{1}$, Shinko K. Lin ${ }^{1}$, Sam H. Ridgway ${ }^{2}$ \\ ${ }^{1}$ Emory University \\ ${ }^{2}$ Navy Marine Mammal Program
}

\section{KEYWORDS}

cerebellum, Tursiops truncates, Delphinus delphis, brain, Odontoceti, comparative neuroanatomy

\begin{abstract}
According to the 'developmental constraint hypothesis' of comparative mammalian neuroanatomy, brain growth follows predictable allometric trends. Therefore, brain structures should scale to the entire brain in the same way across mammals. Evidence for a departure from this pattern for cerebellum volume has recently been reported among the anthropoid primates. One of the mammalian groups that has been neglected in tests of the 'developmental constraint hypothesis' is the cetaceans (dolphins, whales, and porpoises). Because many cetaceans possess relative brain sizes in the range of primates comparative tests of the 'developmental constraint hypothesis' across these two groups could help to delineate the parameters of this hypothesis. In this paper, we compare relative cerebellum volumes in two cetacean species, the bottlenose dolphin (Tursiops truncatus) and the common dolphin (Delphinus delphis), with published data from anthropoid primates. We found that relative cerebellum size is significantly greater in the two dolphin species than in any of the primates, including humans. These results suggest that there is possibly expansion of brain structures independent of strictly allometric processes.
\end{abstract}

\section{Introduction}

According to the 'developmental constraint hypothesis' of comparative mammalian neuroanatomy, mammalian brain growth follows predictable allometric trends [Finlay and Darlington, 1995]. Localized expansion of a single brain structure is unlikely without corresponding enlargement of other structures. In a test of this hypothesis based on a sample of 131 mammalian species, brain size accounted for $96.29 \%$ of the variance in the volumes of component structures [Finlay and Darlington, 1995]. One of these structures was the cerebellum, the size of which correlated at $r=0.996$ [R. Darlington, pers. commun.] with total brain volume. Additional support for these findings comes from postmortem volumetric data suggesting that mammalian cerebella grow allometrically with total brain volume [Radinsky, 1975; Jerison, 1997]. Despite these consistent findings, a more fine-grained analysis restricted to the anthropoid primates revealed departures from allometry, with apes having larger cerebella for their brain size than monkeys [Rilling and Insel, 1998]. These findings suggest that the developmental constraint hypothesis might apply to a broad range of species, but that deviations from its predictions can be found within more restricted phylogenetic ranges. 
One of the mammalian groups absent from the Finlay and Darlington [1995] study is the Cetacea. Cetaceans (dolphins, whales, and porpoises) diverged evolutionarily from terrestrial mammals approximately fifty-five million years ago [Barnes et al., 1985] and have consequently developed a brain that is structurally (and possibly functionally) very different from that of other mammals, particularly in terms of cortical cytoarchitecture [Glezer et al., 1988, 1992], lobular organization [Morgane et al., 1980] and surface morphology [Morgan et al., 1980]. In conjunction with these differences, cetaceans, particularly members of the suborder Odontoceti (toothed whales, dolphins, and porpoises), have evolved encephalization levels comparable to, and in many cases exceeding, those of living anthropoid primates [Marino, 1998]. Specifically, the encephalization level for several dolphin species of the family Delphinidae is second only to modern humans [Marino, 1998]. In addition to this convergence in overall relative brain size with primates, numerous lines of evidence point to similarities between odontocetes and primates in terms of behavioral ecology and social structure [Connor et al., 1992] and cognitive processing and problem solving [Herman, 1986]. Such similarities in encephalization level and cognitivebehavioral characteristics in the face of substantial differences in neuroanatomical organization and evolutionary history suggest that comparisons of cetaceans and primates might be revealing tests of the generalizability of recent hypotheses about brain development and evolution such as the 'developmental constraint hypothesis'. In order to test the applicability of the 'developmental constraint hypothesis' to cetaceans and make more direct comparisons between primates and cetaceans in terms of brain allometry, we examined the relationship between cerebellar and total brain volume (and body size) in the bottlenose dolphin and the common dolphin and compared these data with published data from primates [Rilling and Insel, 1998].

\section{Materials and Methods}

\section{Specimens}

The common dolphin (Delphinus delphis) sample consists of ten (six male, four female) adult postmortem specimens. The bottlenose dolphin (Tursiops truncatus) sample consists of twenty-one (twelve male, nine female) adult postmortem specimens. The dolphin specimens either died in captivity or upon stranding.

The adult primate sample consisted of data from in vivo MRI scans of six humans, four bonobos (Pan paniscus), six common chimpanzees (Pan troglodytes), two gorillas (Gorilla gorilla), four orangutans (Pongo pygmaeus), four gibbons (Hylobates lar), two baboons (Papio cynocephalus), four rhesus monkeys (Macaca mulatta), four sooty mangabeys (Cercocebus atys), four capuchin monkeys (Cebus apella), and four squirrel monkeys (Saimiri sciureus). See Rilling and Insel [1998] for details.

\section{Weight Measurements}

Upon death, the brains of the dolphins were removed from the skull and immersed in $10 \%$ buffered formalin. (Given the similar proportions of gray and white matter in the cerebellum and cerebrum, there is no reason to suspect that fixation causes differential shrinkage in the two structures.) The total body length and weight from facility records were obtained for all of the specimens. Total fixed brain and cerebellum weights were obtained using an electronic balance. In order to compare the dolphin weight measurements with the volumetric data for the primate sample in Rilling and Insel (1998) whole brain and cerebellum weights were converted to volumetric units by dividing the weights by the specific gravity of brain tissue or 1.036 [Stephan et al., 1981].

\section{Statistical Analysis}

We examined relative cerebellum size in dolphins and primates with several analyses. First, in order to obtain an initial straightforward value of the percentage of total brain occupied by the cerebellum for each 
of the groups, a ratio of cerebellum to total brain volume was calculated for the dolphin sample and compared with the same ratio for the primate sample. Second, log cerebellum volume was regressed on log 'total brain minus cerebellum' volume (hereonin referred to as 'noncerebellar brain volume') for the dolphins, apes, monkeys, and humans separately and a line was fitted through each sample using the method of least squares. We plotted cerebellum volume versus noncerebellar brain volume, rather than plotting cerebellum versus total brain volume in order to avoid including the $y$ variable as part of the $x$, which would render the analysis less sensitive to detecting departures from allometry [Deacon, 1990]. A test of the General Linear Model was conducted to determine whether there were significant differences in the regression slopes and intercepts among groups that would indicate the presence of group differences in scaling factors (slopes) and/or elevations (intercepts). The following general linear model was used:

Log cerebellum $=\beta_{0}+\beta_{1} \log$ (total brain - cerebellum $)+\beta_{2}$ Taxa $+\beta_{3} \log$ (total brain cerebellum) * Taxa, where taxa is a dummy variable that takes on values of 1 and 0 .

Pairs of taxa were selected and one taxon was assigned a value of taxa $=0$ and the other taxa $=1$. If $\beta_{3}$ (the interaction term) is significant in the model, then the slopes of the regression lines for the two taxa should differ. Otherwise, $\beta_{3}$ is dropped from the model and $\beta_{2}$ tests for a significant difference in elevation ( $y$ intercept after fitting a common slope) of the regression lines for the two taxa. We constructed six models to test all pairwise comparisons: dolphin vs. monkey; dolphin vs. ape; dolphin vs. human; ape vs. monkey; human vs. monkey; human vs. ape.

The size of the cerebellum relative to the rest of the brain in humans is smaller than in apes [Rilling and Insel, 1998]. However, this result is somewhat misleading in that it probably reflects the marked enlargement of the forebrain in humans. An analysis of cerebellar volume relative to body size clarifies the situation. Relative to body size, humans have a larger cerebellum than apes [Rilling and Insel, 1998]. The two dolphin species in the present study also exhibit marked elaboration of cortical areas [Morgane et al., 1980] which might similarly confound relative cerebellum size results. Therefore, log cerebellum volume was regressed on log body weight for the dolphins, apes, monkeys, and humans separately and a line was fitted through each sample using the method of least squares. A test of the General Linear Model was conducted to determine whether there were significant differences in the regression slopes and intercepts among groups that would indicate the presence of group differences in scaling factors and/or elevations. The following general linear model was used:

Log cerebellum $=\beta_{0}+\beta_{1} \log$ (body weight) $+\beta_{2}$ Taxa $+\beta_{3} \log$ (body weight) * Taxa, where taxa is a dummy variable that takes on values of 1 and 0 .

Pairs of taxa were selected and one taxon was assigned a value of taxa $=0$ and the other taxa $=1$. If $\beta_{3}$ (the interaction term) is significant in the model then the slopes of the regression lines for the two taxa are different. Otherwise, $\beta_{3}$ is dropped from the model and $\beta_{2}$ tests for a significant difference in elevation (y intercept after fitting a common slope) of the regression lines between the two taxa. We constructed three models to test the following pairwise comparisons: dolphin vs. monkey; dolphin vs. ape; ape vs. monkey.

Finally, it should be noted that the comparisons in the present study are not always taxonomically equivalent in that each family is represented by a different numbers of species, e.g. two dolphin species vs. one human species.

However, all of the comparisons are made at a between-family level, which should strengthen the consistency of the results. 


\section{Results}

In order to visually compare the proportions of a dolphin cerebellum with that of a primate cerebellum, figure 1 displays sagittal Magnetic Resonance Imaging-based slices through one hemisphere of a living human $(a-f)$ and a postmortem bottlenose dolphin $(g-l)$ brain. The bottlenose dolphin cerebellum is clearly larger in relation to the rest of the brain than is the human cerebellum.

Table 1 displays the cerebellum volume, brain volume, and body weight of the thirty-one dolphin specimens in the study. Table 2 displays the mean, standard deviation and upper and lower 95\% confidence intervals of cerebellum and total brain volume for the bottlenose dolphin and the common dolphin group. There are no differences in relative cerebellum size (as measured by the ratio of the cerebellum to total brain volume) between the common dolphin and bottlenose dolphin. Therefore, all data for the two species were combined.

Figure 2 displays a box and whiskers plot of the ratio of cerebellum volume to total brain volume for the dolphin sample and the major primate groups. An analysis of variance (ANOVA) revealed that the mean ratios for the groups differ significantly $(F=51.61$, $d f=5,69 ; p<0.0001)$. A Tukey post-hoc test showed that the mean ratio for the dolphins $(15.1 \%)$ is significantly greater than all of the primate means $(10.3 \%$ for humans, $13.0 \%$ for gibbons, $13.3 \%$ for great apes, $9.2 \%$ for Cercopithecidae, i.e. baboons, rhesus, and mangabeys, and $9.3 \%$ for Cebidae, i.e. cebus and squirrel monkeys, all $p$ values $<0.037$ ). The post hoc test also replicated the finding that the means for the apes (gibbons and great apes) are significantly higher than the means for the monkeys and humans (all $p$ values $<0.027$ ). Figure 2 graphically shows that, at $15.1 \%$ of the total brain volume, the cerebellum in the dolphins is significantly larger in relative size than it is in any of the primates. The large relative cerebellum size of great apes and gibbons compared with humans and monkeys is also evident.

In order to investigate whether the allometric relationship between the size of the cerebellum and the rest of the brain is similar in all of the groups, separate regression analyses of log cerebellar volume on the log of noncerebellar brain volume for the dolphin and each of the primate groups were conducted. Figure 3 graphically displays the results of a least squares regression of log cerebellum volume on the log of noncerebellar brain volume for the dolphin group and each of the primate groups separately. Separate regression lines were fitted through the ape (gibbon and great apes), dolphin, human, and monkey groups respectively. The equation describing the dolphin data is $Y=0.97 X-0.68$. The equation describing the ape data is $Y=1.01 X-0.86$. The human data are characterized by $Y=0.98 X-0.88$ and the monkey data by $Y=1.04 X-1.08$. The general linear model test for significant differences in slopes and intercepts for all pairwise comparisons (dolphin vs. monkey; dolphin vs. ape; dolphin vs. human; ape vs. monkey; human vs. monkey; human vs. ape) revealed that there are no significant differences in the slopes of the regression equations for any of the groups. However, there are significant differences among a number of the groups in terms of their elevation (intercept after fitting a common slope) (all $p$ values < 0.015). These differences indicate that, after controlling for brain size, dolphins and primates differ in relative cerebellar volume. These differences can be quantified by obtaining the anti-log of $\beta_{2}$. After controlling for brain size, the average dolphin cerebellum is $17.2 \%$ larger than the average ape cerebellum, 53.5\% larger than the average human cerebellum, and $67.5 \%$ larger than the average monkey cerebellum. Comparisons among the primates reveal that the ape cerebellum is $34 \%$ larger than the human cerebellum and $\mathbf{4 5 . 2 \%}$ larger than the monkey cerebellum, controlling for brain size. There is no significant difference in elevation between humans and monkeys.

Another way to test for differences in relative cerebellum size between dolphins and primates is to use primate regression lines to predict dolphin cerebellum size from dolphin total brain size and then test for differences between the predicted and observed dolphin cerebellar volumes. Observed and predicted 
(from the primate regression line) cerebellar volumes from the sample of thirty-one dolphins are then compared using a paired t-test and the average difference between observed and predicted cerebellum volumes can be examined. With this method, the average dolphin cerebellum is significantly larger by $15.5 \%$ than predicted for an ape of the same noncerebellar brain volume $(t=6.940, d f=30, p<0.0001)$. The average dolphin cerebellum is significantly larger, by $55.4 \%$, than predicted for a monkey of the same noncerebellar brain volume $(t=14.675, \mathrm{df}=30, \mathrm{p}<0.0001)$. Finally, the average dolphin cerebellum is significantly larger, by $49.5 \%$, than predicted for a human of the same noncerebellar brain volume $(\mathrm{t}=$ 13.253, $\mathrm{df}=30, \mathrm{p}<0.0001)$. Therefore, both the regression model and the 'observed versus predicted' tests converge upon similar results for differences among the taxa in the relationship between cerebellar size and the size of the rest of the brain.

Fig. 1. Sagittal Magnetic Resonance Imaging-based slices through one hemisphere of a living human (a-f) and a postmortem bottlenose dolphin (g-l) brain. In both brains images span the entire medial to lateral extent of the cerebellum. The $1.2 \mathrm{~mm}$ thick human slices (a-f) are $0 \mathrm{~mm}, 10.8 \mathrm{~mm}, 21.6 \mathrm{~mm}, 32.4 \mathrm{~mm}, 43.3 \mathrm{~mm}$, and $54 \mathrm{~mm}$ lateral of the midsagittal plane, respectively. The $2 \mathrm{~mm}$ thick dolphin slices $(\mathbf{g}-\mathbf{l})$ are $0 \mathrm{~mm}, 12 \mathrm{~mm}, 24 \mathrm{~mm}, 36 \mathrm{~mm}, 48 \mathrm{~mm}$, and $60 \mathrm{~mm}$ lateral of the midsagittal plane, respectively. Total brain volume for the human is $1330.00 \mathrm{~cm} 3$ and for the dolphin $1420.50 \mathrm{~cm} 3 . \mathrm{C}=$ Cerebellum.
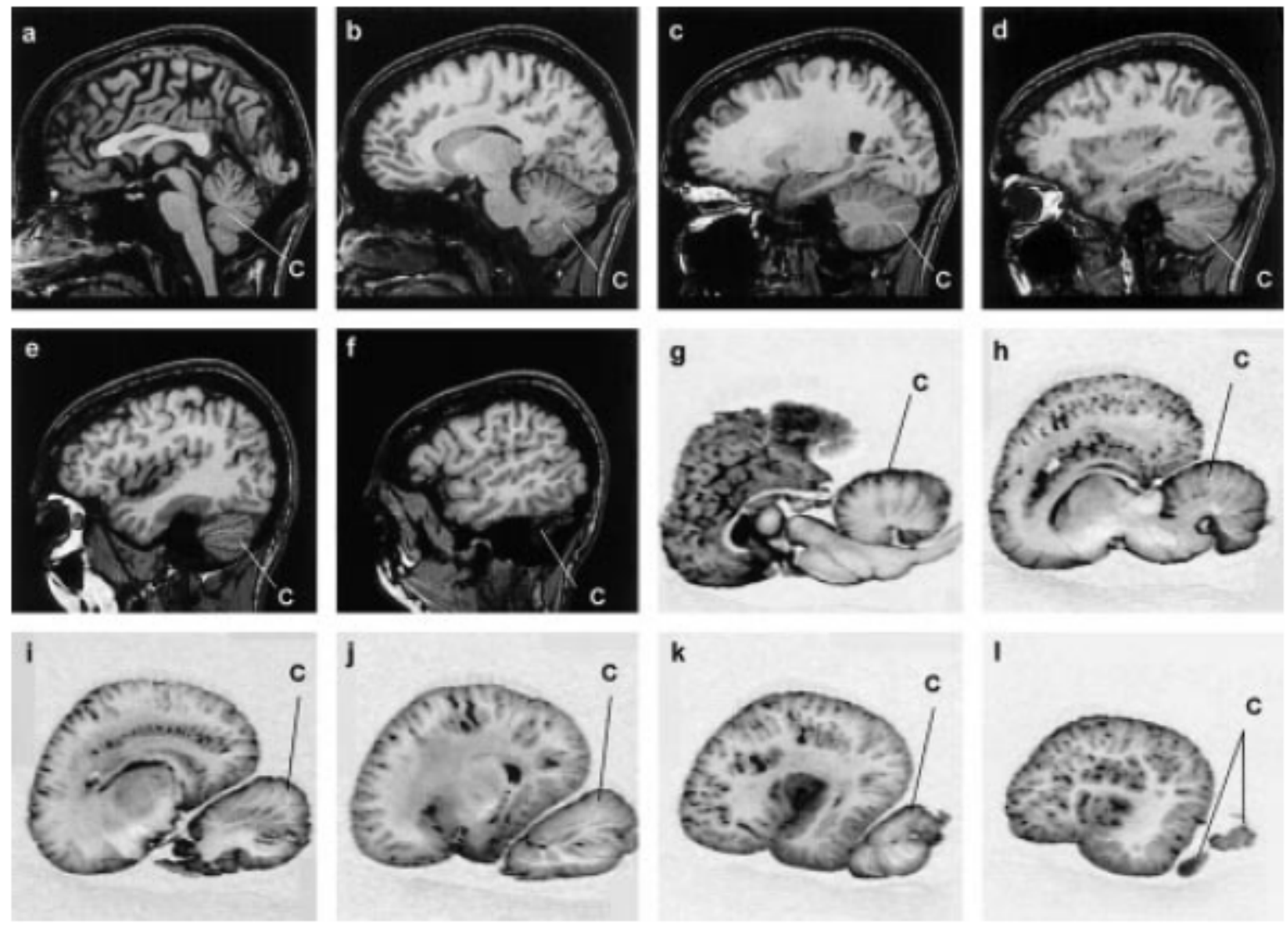

In order to investigate the relationship between cerebellum volume and body size, log cerebellum volume was regressed on log body weight for the dolphins, apes, monkeys, and humans separately and a line was fitted through each sample using the method of least squares. Figure 4

graphically displays the results of a least squares regression of log cerebellum volume on log body weight for the dolphin group and each of the primate groups separately. The equation describing the dolphin data is $Y=0.68 X-0.53$. The equation describing the ape data is $Y=0.60 X-0.60$. The monkey data are 
characterized by $Y=0.53 X-0.67$. In terms of encephalization level, that is, brain size in relation to body size when brain-body allometry is taken into account, dolphins are surpassed only by humans [Marino, 1998]. However, figure 4 shows that, in terms of relative cerebellar size (cerebellum size relative to body size), dolphins and humans are equivalent.

Table 1. Cerebellum volume, brain volume, and body weight of the thirty-one dolphin specimens in the study

\begin{tabular}{|c|c|c|}
\hline Cerebellum volume $\left(\mathrm{cm}^{3}\right)$ & Brain volume $\left(\mathrm{cm}^{3}\right)$ & Body weight (kg) \\
\hline \multicolumn{3}{|l|}{ Common dolphin } \\
\hline 107.143 & 726.834 & 59 \\
\hline 125.483 & 780.888 & 86 \\
\hline 120.656 & 779.923 & 58 \\
\hline 92.664 & 664.093 & 55 \\
\hline 136.100 & 990.347 & 86 \\
\hline 114.865 & 845.560 & 89 \\
\hline 131.274 & 755.792 & 75 \\
\hline 116.795 & 730.695 & 91 \\
\hline 116.795 & 783.784 & 75 \\
\hline 123.552 & 805.019 & 90 \\
\hline \multicolumn{3}{|l|}{ Bottlenose dolphin } \\
\hline 250.000 & 1614.865 & 151 \\
\hline 223.938 & 1323.359 & 132 \\
\hline 224.903 & 1503.861 & 177 \\
\hline 273.166 & 1500.965 & 195 \\
\hline 208.494 & 1361.004 & 226 \\
\hline 213.320 & 1561.776 & 156 \\
\hline 215.251 & 1395.753 & 164 \\
\hline 230.695 & 1572.394 & 172 \\
\hline 218.147 & 1453.668 & 161 \\
\hline 248.069 & 1664.093 & 198 \\
\hline 290.541 & 1843.629 & 245 \\
\hline 213.320 & 1548.263 & 178 \\
\hline 264.479 & 1783.784 & 186 \\
\hline 183.398 & 1408.301 & 196 \\
\hline 256.757 & 1568.533 & 152 \\
\hline 197.876 & 1552.124 & 170 \\
\hline 214.286 & 1430.502 & 136 \\
\hline 215.251 & 1471.042 & 170 \\
\hline 222.008 & 1632.239 & 268 \\
\hline 288.610 & 1716.216 & 222 \\
\hline 217.181 & 1456.564 & 190 \\
\hline
\end{tabular}


Table 2. The mean, standard deviations, and upper and lower $95 \%$ confidence intervals of cerebellum and total brain volume for the bottlenose dolphin and the common dolphin groups

\begin{tabular}{|lll|}
\hline & Mean total brain volume $\left.\mathbf{( c m}^{3}\right)$ & Mean cerebellum volume $\left.\mathbf{( c m}^{\mathbf{3}}\right)$ \\
\hline \multirow{2}{*}{ Common dolphin } & $786.29(86.97)$ & $118.53(12.33)$ \\
& $(724.08,848.51)$ & $(109.72,127.35)$ \\
Bottlenose dolphin & $1541.09(135.25)$ & $231.89(29.03)$ \\
& $(1479.53,1602.66)$ & $(218.68,245.11)$ \\
\hline
\end{tabular}

Fig. 2. Box and whiskers plot of the ratio of cerebellum volume to total brain volume for the dolphin specimens (= Delph) and the primate groups from Rilling and Insel [1998]. Cebid = Cebidae, Cerco = Cercopithecidae, Pongid $=$ Pongidae, Human, Hylobat $=$ Hylobatidae.

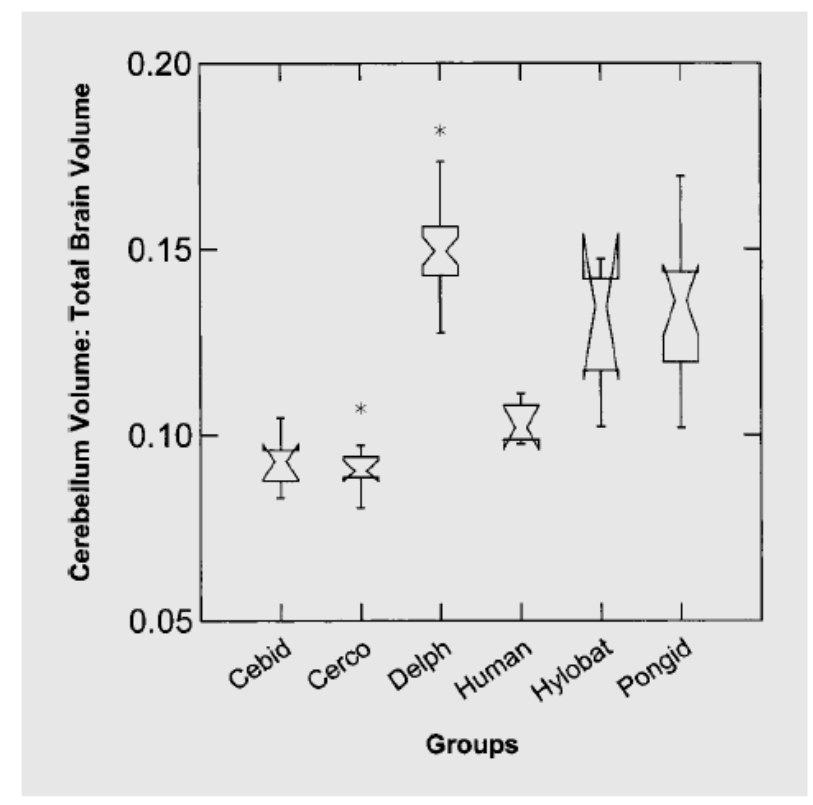

Fig. 3. Separate least squares regression lines for log cerebellum volume on log noncerebellar volume for the dolphin, ape (Pongidae and Hylobatidae), human, and monkey (Cercopithecidae and Cebidae) groups.

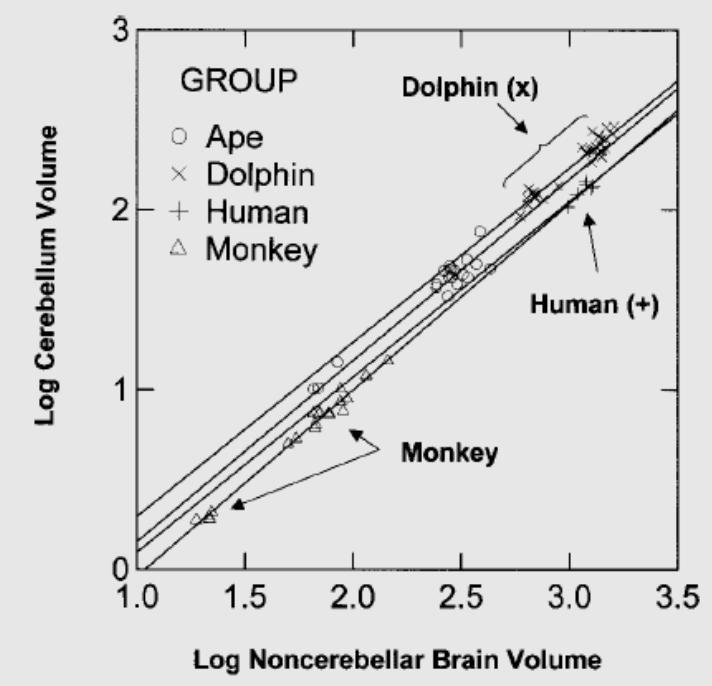


Fig. 4. Separate least squares regression lines for log cerebellum volume on log body weight for the dolphin, ape, human, and monkey groups.

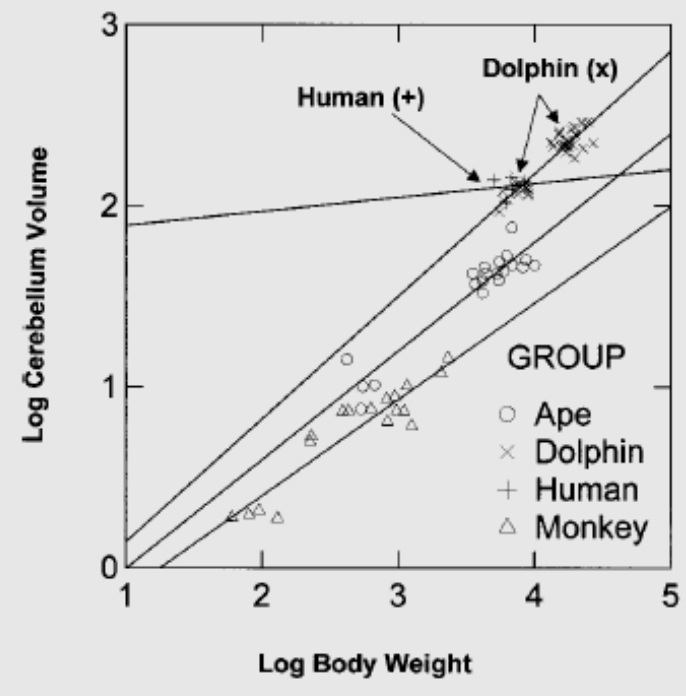

Figure 4 also shows that the human data cannot be meaningfully described as a regression equation because it is comprised of only one species in which there is an insufficient range of body size values on which to base the function. Therefore, the human data were omitted from the following analysis. The general linear model test for significant differences in slopes and intercepts for the following pairwise comparisons (dolphin vs. monkey; dolphin vs. ape; ape vs. monkey) revealed no significant differences in the slopes of the regression equations for any of the groups. There is a marginally significant difference between the slopes for dolphins and monkeys $(t=1.731, \mathrm{df}=30, \mathrm{p}=0.09)$, but this effect did not account for more than $2 \%$ of the variance. However, there are significant differences among a number of the groups in terms of the intercept after fitting a common slope (all $p$ values $<0.0001$ ). These differences indicate that, after controlling for body size, dolphins and primates differ in relative cerebellar volume. These differences can be quantified by obtaining the anti-log of b2. After controlling for body size, the average dolphin cerebellum is $139 \%$ larger than the average ape cerebellum and $386 \%$ larger than the average monkey cerebellum. (Note that any shrinkage due to fixation of the dolphin cerebellum would have biased us against detecting a disproportionately large dolphin cerebellum.) Finally, comparisons among the primates reveals that the ape cerebellum is $89.2 \%$ larger than the monkey cerebellum.

We calculated expected values for cerebellum volume in dolphins based on the primate regression functions and statistically tested for differences between observed and expected values in the dolphins. With this method, the average dolphin cerebellum is significantly larger, by $149 \%$, than predicted for an ape of the same body weight $(t=15.074, d f=30, p<0.0001)$. The average dolphin cerebellum is significantly larger, by $476 \%$, than predicted for a monkey of the same body weight $(t=17.023, \mathrm{df}=30, \mathrm{p}$ $<0.0001$ ). Therefore, both the regression model and the 'observed versus predicted' tests converge upon similar results for differences among the taxa in the relationship between cerebellum and body size.

\section{Discussion}

The present results demonstrate that dolphin cerebella, which average about $15 \%$ of total brain size, are significantly larger than that of human and nonhuman anthropoid primates after controlling for brain 
volume. Therefore, although the 'developmental constraint hypothesis' is broadly accurate [Finlay and Darling, 1995] our analysis shows that the relationship between the cerebellum and brain size in primates cannot be used to predict cerebellum size in dolphins.

What is the function of the large cerebellum in dolphins? The cerebellum has traditionally been viewed as having a singular role in the control and coordination of movements. Recently, evidence points to a broader function for the cerebellum that includes involvement in sensory processing [Paulin, 1993], memory [Desmond et al., 1997; Andreasen et al., 1999], cognition [Leiner et al., 1993, 1995; Fiez, 1996; Allen et al., 1997], and, in humans, language [Leiner et al., 1993]. Paulin [1993] has speculated that the cerebellum is best characterized as a dynamic system for tracking the flow of incoming and outgoing sensory and motor information. This kind of 'dynamical state estimation' is not unlike the kind of processing that occurs during echolocation. In echolocation high frequency sound waves are emitted by the animal and the echoes from these emissions are picked up and rapidly processed in acoustic brain structures. Recent findings have shown that the cerebellum might be intimately involved in acoustic timing. For example, cerebellar neurons function in circadian timing [Fauteck et al., 1994] and in sound location in the big brown bat, Eptesicus fuscus [Kamada and Jen, 1990] and in the acoustics of the biosonar signals and frequency sensitivity in the mustached bat Pteronotus parnelli [Horikawa and Suga, 1986]. In reviewing the evidence, Ridgway [2000] has suggested that the cerebellum is involved in acoustic processing in delphinoids, among other functions. The regions of the cerebellum that are particularly expanded in odontocetes (echolocating dolphins, porpoises, and whales) as revealed by qualitative examination are the paramedian lobules and paraflocculus [Breathnach, 1960; Jansen and Jansen, 1969; Ridgway, 1990; Paulin, 1993]. These regions receive sensory inputs used in spatial orientation, navigation, prey detection, and communication [Paulin, 1993]. These are all functions served by echolocation. These areas are more expanded in echolocating bats than in non-echolocating bats [Henson, 1970]. Also, vermal lobule VIII is more highly developed in echolocating odontocetes than in the non-echolocating baleen whales and expanded in echolocating bats [Paulin, 1993]. This has led Paulin [1993] to suggest that vermal lobule VIII is involved in echolocation.

It should be noted, however, that non-echolocating baleen whales also possess relatively large cerebella [Ridgway, 1990; Paulin, 1993]. However, in echolocating odontocetes the particular areas of the cerebellum involved in auditory processing are specifically expanded. Taken together, the evidence suggests that the large dolphin cerebellum could be a result of selection for rapid dynamic tracking added to considerable motor control. In accordance with the hypothesis that the cerebellum is associated with a versatile array of functions, Rilling and Insel [1998] point out that one of the largest relative cerebellum values among the anthropoid primates is found in the gibbon, which has one of the most versatile locomotor repertoires [Hollihn, 1984]. It could be that, as Paulin [1993] suggests, the cerebellum is not involved solely in locomotion, but rather the dynamical coordination of both sensory and motor inputs and outputs. If this is the case then one could argue that other mammalian species that rely heavily on fast dynamic tracking, such as felid predators or pinnipeds, would also display enlargement of either the whole cerebellum or those parts of the cerebellum most involved in dynamic sensory-motor integration. These issues can be clarified in the future by analyses of a broader number of species and, in particular, studies of the separate subcomponents of the cerebellum to determine which structures contribute most to the size of the cerebellum in different species.

In conclusion, the present findings suggest that there are deviations from a strictly allometric relationship between the brain and its components among mammals. These deviations shape the allometry in ways that might reflect species-specific selection pressures. Independent variation of individual brain parts is not inconsistent with the kind of correlated brain growth discussed by Finlay and Darlington [1995]. The non-allometric variation in brain part size that characterizes our findings can be conceptualized as a 
species-specific refinement of perhaps a larger allometric pattern. Dolphin relative cerebellum size is a particularly instructive example of this pattern.

\section{Acknowledgements}

The authors wish to thank Dr. Irwin Waldman and Dr. Scott Lilienfeld of Emory University for statistical advice. We also thank Dr. James Gilchrest of the National Marine Fisheries Service and Dr. Raymond J. Tarpley of Texas A \& M University for help in collecting the dolphin material. This work was made possible by the Yerkes Regional Primate Research Center and NIH grants RR-00165 and P51-0169I, as well as grants from the L.S.B. Leakey Foundation and the Markey Foundation for Neurological Sciences to Jim Rilling.

\section{References}

Allen, G., R.B. Buxton, E.C. Wong, and E. Courchesne (1997) Attention activation of the cerebellum independent of motor involvement. Science, 275: 1940-1943.

Andreasen, N.C., D.C. O' Leary, S. Paradiso, T. Cizadlo, S. Arndt, G.L. Watkins, L. Boles Ponto, and R.D. Hichwa (1999) The cerebellum plays a role in conscious episodic memory retrieval. Human Brain Mapping, 8: 226-234.

Barnes, L.G., D.P. Domning, and C.E. Ray (1985) Status of studies on fossil marine mammals. Marine Mammal Sci., 1: 15-53.

Breathnach, A.S. (1960) The cetacean central nervous system. Biol. Rev., 35: 187-230.

Connor, R.C., R.A. Smolker, and A.F. Richards (1992) Dolphin alliances and coalitions. In Coalitions and Alliances in Humans and Other Animals (ed. by A.H. Harcourt and F.B.M. DeWaal), Oxford University Press, Oxford, UK, pp. 415-443.

Deacon, T.W. (1990) Rethinking mammalian brain evolution. Am. Zool., 30: 629-705.

Desmond, J.E., J.D.E. Gabrieli, A.D. Wagner, B.L. Ginier, and G.H. Glover (1997) Lobular patterns of cerebellar activation in verbal workingmemory and finger-tapping tasks as revealed by functional MRI. J. Neurosci., 17: 9675-9685.

Fauteck, J.D., A. Lerchl, M. Bergmann, M. Moller, F. Fraschini, W. Wittkowski, and B. Stankov (1994) The adult human cerebellum is a target of the neuroendocrine system involved in circadian timing. Neurosci. Lett., 179: 60-64.

Fiez, J.A. (1996) Cerebellar contributions to cognition. Neuron, 16: 13-15.

Finlay, B.L, and R.B. Darlington (1995) Linked regularities in the development and evolution of mammalian brains. Science, 268: 1578-1584.

Glezer, I.I., P.R. Hof, C. Leranth, and P.J. Morgane (1992) Morphological and histological features of odontocete visual neocortex: immunocytochemical analysis of pyramidal and nonpyramidal populations of neurons. In Marine Mammal Sensory Systems (ed. by J.A. Thomas, R.A. Kastelein, and A.Y. Supin), Plenum Press, New York, N.Y., pp. 1-38.

Glezer, I.I., M.S. Jacobs, and P.J. Morgane (1988) Implications of the 'initial brain' concept for brain evolution in Cetacea. Behav Brain Sci., 11: 75-116. 
Henson, O.W. (1970) The central nervous system. In Biology of Bats, Vol. II (ed. by W.A. Wimsatt), Academic Press, London, UK, pp. 57-152.

Herman, L.M. (1986) Cognition and language competencies of bottlenosed dolphins. In Dolphin Cognition and Behavior: A Comparative Approach (ed. by R.J. Schusterman, J.A. Thomas, and F.G. Wood), Lawrence Erlbaum, Hillsdale, N.J., pp. 221-252.

Hollihn, U. (1984) Bimanual suspensory behavior: morphology, selective advantages and phylogeny. In The Lesser Apes (ed. by H. Preushoft, D.J. Chivers, W.Y. Brockelman and N. Creel), Edinburgh University Press, Edinburgh, UK, pp. 85-95.

Horikawa, J., and N. Suga (1986) Biosonar signals and cerebellar auditory neurons of the mustached bat. J. Neurophysiol., 55: 1247-1267.

Jansen, J., and J.K.S. Jansen (1969) The nervous system of Cetacea. In Biology of Marine Mammals (ed. by H.T. Andersen), Academic Press, New York, N.Y., pp. 175-252.

Jerison, H.J. (1997) Evolution of prefrontal cortex. In Development of the Prefrontal Cortex: Evolution, Neurobiology, and Behavior (ed. By N.A. Krasnegor, G.R. Lyon, and P.S. Goldman- Rakic), Paul H. Brookes, Baltimore, Md., pp. 9-26.

Kamada, T., and P.H. Jen (1990) Auditory response properties and directional sensitivity of cerebellar neurons of the echolocating bat, Eptesicus fuscus. Brain Res., 528: 123-129.

Leiner, H.C., A.L. Leiner, and R.S. Dow (1993) Cognitive and language functions of the human cerebellum. Trends Neurosci., 16: 444-447.

Leiner, H.C., A.L. Leiner, and R.S. Dow (1995) The underestimated cerebellum. Human Brain Mapping, 2: 244-254.

Marino, L. (1998) A comparison of encephalization between odontocete cetaceans and anthropoid primates. Brain Behav. Evol., 51: 230-238.

Morgane P.J., M.S. Jacobs, and W.L. MacFarland (1980) The anatomy of the brain of the bottlenose dolphin (Tursiops truncatus). Surface configurations of the telencephalon of the bottlenose dolphin with comparative anatomical observations in four other cetacean species. Brain Res. Bull., 5: 1-107.

Paulin, M.G. (1993) The role of the cerebellum in motor control and perception. Brain Behav. Evol., 41: $39-50$.

Radinsky, L. (1975) Primate brain evolution. Am. Sci., 63: 656-663.

Ridgway S.H. (1990) The central nervous system of the bottlenose dolphin. In The Bottlenose Dolphin (ed. by S. Leatherwood and R. Reeves), Academic Press, San Diego, Calif., pp. 69-97.

Ridgway, S.H. (2000) The auditory central nervous system of dolphins. In Hearing in Whales and Dolphins (ed. by W. Au, A. Popper, and R. Fay), Springer Verlag, New York, N.Y., pp. 273-293.

Rilling, J.K., and T.R. Insel (1998) Evolution of the cerebellum in primates: differences in relative volume among monkeys, apes and humans. Brain Behav. Evol., 52: 308-314. 
Stephan, H., H. Frahm, and G. Baron (1981) New and revised data on volumes of brain structures in insectivores and primates. Folia Primatol., 35: 1-29. 